\title{
Infrared thermography as an objective technique for evaluation of patch tests
}

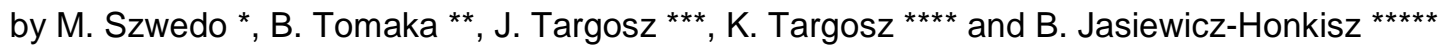 \\ * MONIT SHM Sp. z o.o., 34 Lublanska St., 31-476 Krakow, Poland, mszwedo@monitshm.pl \\ ** AGH-University of Science and Technology, 30 Mickiewicza Av., 30-059 Krakow, Poland, \\ tomaka@agh.edu.pl \\ *** AGH-University of Science and Technology, 30 Mickiewicza Av., 30-059 Krakow, Poland, \\ jantargosz@interia.pl

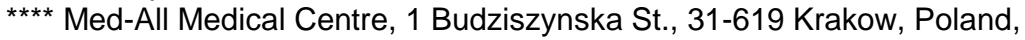

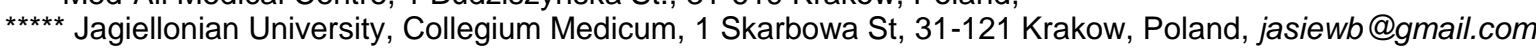

\begin{abstract}
Skin test is a method of medical diagnosis that attempts to provoke a small, controlled, allergic response. Evaluation of the patch test depends on visual assessment so it is not an objective method, because it does concern only skin surface and difficulties like changes in skin pigmentation, atopic skin, black race skin or delayed reaction may occur. Studies with use of thermography confirm that skin surface allergic reaction is strictly connected with temperature increasing at that area so such technique may be the key for impartial evaluation. Carried out tests, also confirmed information from literature review, that large intervals between allergens must be kept.
\end{abstract}

\section{Introduction}

Dermatological diagnostic of dermatitis and allergy diagnostics in many cases is still based on subjective techniques of evaluation. In allergology the clinical evaluation of skin test reaction is achieved by visual impression combined with measurements of the intensity of the reaction on the usual scale of differentiation. In this paper authors presents results of carried out tests with the use of infrared thermography which were undertake to check out if it can improve assessment of commonly used non-objective techniques of evaluating patch tests.

Thermography is a valuable, non-contact measurement technique, which can find practical application in dermatological diagnostics, but only if all standardisation rules are adhered, appropriate terms and conditions in consulting room and patient's preparation among other things. Every negligence in testing procedure may produce an effect that may distort a result, and make it useless. It can be useful not only in diagnosis of skin tumours, particularly melanoma, or burn diagnostics, but also in allergy diagnosis, which is more and more common disease.

According to [1][2][3] the essence of the thermography is to compare temperature of skin with positive reaction to allergen with temperature of surrounding healthy skin, so information about gradient of temperature is more important than its absolute value. Maximal gradient for the strongest allergic reactions is within 2 and $3^{\circ} \mathrm{C}$ (accuracy of measurement depends on parameters of used camera).

Skin tests are commonly used in allergic diagnostics. It is a method of medical diagnosis that attempts to provoke a small, controlled, allergic response. In patch test, which are used for diagnostics of allergic contact dermatitis, strips of tape containing small quantities of common allergens are applied onto the skin, usually on the back, for forty eight hours incubation. The skin reaction is observed at regular intervals, usually after 2, 3 and 4 days. Evaluation of the test depends only on visual assessment. It is not an objective method, because it does concern only skin surface and difficulties like changes in skin pigmentation, atopic skin, black race skin or delayed reaction may occur. Additional reading after 7 days might disclose another positive reactions for allergens which were negative in previous readings. Patch test diagnostics reduces costs of treatment and improves quality of life because it enables and shorten final diagnosis. Results of such tests help to avoid identified allergic haptens, thus help reduce symptoms, so it is important to do not omit delayed reactions. [4][5]

Allergic reactions occur when a person's immune system reacts to normally harmless substances in the environment (allergens). This reaction results in an inflammatory response with release of proinflammatory cytokines increase permeability of blood vessels and with typical local symptoms like redness, increase temperature and oedema. Studies with use of thermography confirm that skin surface allergic reaction is strictly connected with temperature increasing in that area. Carried out tests also confirmed information from the literature review, that large intervals, like 8 to $10 \mathrm{~cm}$, between allergens must be kept. [1][2]

Recently there is more and more publications about thermography as an additional technique for patch tests to increase the index of properly diagnosed allergic reactions. For example in cases described in [2] the evaluation of patch test was performed 30 minutes after patch removal. Positive reactions to allergen varied from "+" to "++++" (according to criteria of the International Contact Dermatitis Research Group), and in thermograph was shown as hot area which extended beyond the visible borders of the patch site and was asymmetrically oriented towards the nearest lymph node, spreading as one or more hyperthermic lines. That is the reason to keep large intervals between patches, like 8 to $10 \mathrm{~cm}$. It was also established that temperature and quantity of applied allergens have influence on the temperature 
of tested skin surface, so solutions should be heated up to the ambient temperature (about $20-23^{\circ} \mathrm{C}$ ) and applied in small quantities.

\section{Diagnostic method and standardization}

Patch testing is the only useful and reliable method for identification of an allergic contact dermatitis - it is acknowledged as the gold standard. The International Contact Dermatitis Research Group (ICDRG) was formed to promote the understanding of contact dermatitis and it has had major roles in the standardisation of patch testing for over forty years. [5]

Infrared thermography appeared as an diagnostic technique in medicine at the early 1960 s and first publications on standardization of thermal imaging were published in the 1970s. Few years ago the International Standard Organization has published two new standards to define the use of a thermography in fever screening. The first one, IEC 80601-2-59:2008, describes particular requirements for the basic safety and essential performance of screening thermography for human febrile temperature screening, and the second one, ISO/TR 13154:2009, defines deployment, implementation and operational guidelines for identifying febrile humans using a screening thermograph. However it is about fever screening it provides some guidelines for application of thermal imaging in clinical medicine. These documents dictate standards in manufacture and performance and are specific in the description of calibration procedures. [4][7][9][10]

Emissivity of biological tissues is within 0.85 and 0.98 . For human skin it is reported to be equal $0.98 \pm 0.01$ for wavelength range of $2-20 \mu \mathrm{m}$, it peaks around $10 \mu \mathrm{m}$, regardless of skin colour. Generally, for medical applications narrower wavelength band is used, from 8 to $12 \mu \mathrm{m}$. [1]

\subsection{Patch test - procedure, evaluation and diagnosis}

Patch testing is performed as follows: strips of tape containing small quantities of common allergens are applied onto the skin, usually on the back or on the arm, and it must remain in place and be kept dry for 48 hours. At this time the surface of application should not be rubbed by, for example, an underwear or be scratched in case of false positive reactions. The patient should not take any cortisone medication during the test and avoid exposure of the back to the sun. After that time patches are removed and after several minutes initial reading is performed. After another 1 or 2 days final reading is made. At Fig. 1 the procedure of applying patch tests is presented.

a)

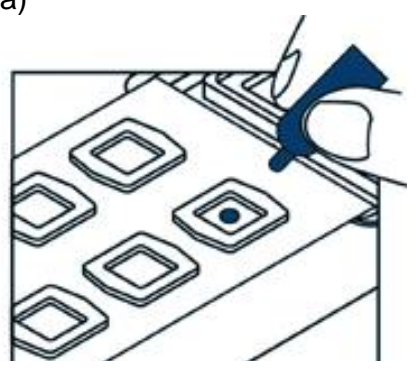

b)

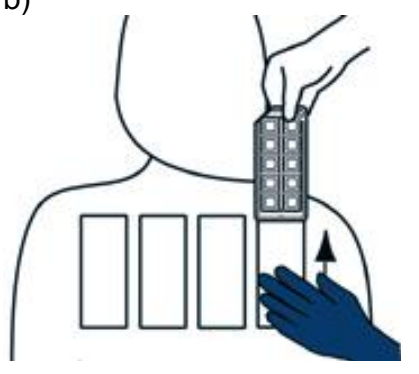

c)

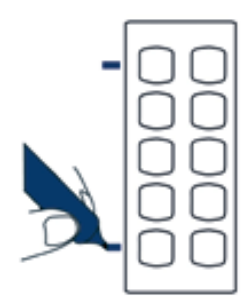

d)

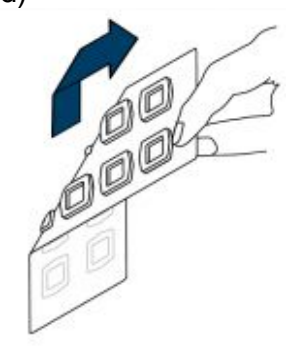

e)

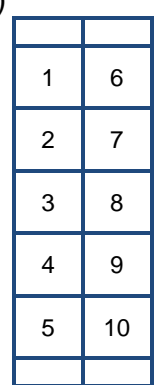

Fig. 1. Applying patch test procedure: a) applying the test preparations, b) applying the unit of chambers onto the back, c) marking chambers, d) removing the patches after 48 hours, e) Arrangement of allergens on the back for strip with 10 chambers [12]

Evaluation of test results is based on visual scheme. It is proceeded by physician on the basis of visual observation. Positive reaction at initial reading is classified as irritant contact dermatitis, while at further readings positive reaction is classified as allergic contact dermatitis (ACT).

\subsection{Standardization}

IR camera should be mounted perpendicularly to the examined surface, emissivity should be settled at 0,98 and sensitivity and accuracy should be defined.[1] Consulting room has to fulfil the following requirements: ambient temperature within $20-24^{\circ} \mathrm{C}$, air humidity within $45-55 \%$, lack of external heat and radiation sources, no draught and right space. Those parameters have to be written down in the test record form.

In case of thermal imaging important part of examination is patient's preparation. It is not allowed to apply substances, medications or drugs and to practise physical exercises even for a few days before test application. Before readings patient should come calmly and for 15 to 30 minutes stay in rest at waiting room. Then acclimatization in consulting room with undressed parts of the body, which will be studied, takes from 5 to 30 minutes. Such rules have to provide better accuracy and repeatability of the measurements.[6][7][8] 
The International Contact Dermatitis Research Group for interpretation of patch test results recommends to use descriptive interpretation scale. Example of such scale is presented at Table 1 and its visual scheme at Fig. 2.

Table 1. Descriptive interpretation scale [5]

\begin{tabular}{|c|l|}
\hline Grade & Meaning / appearance \\
\hline- & Negative reaction \\
\hline $\mathrm{R}$ & Irritant reaction \\
\hline$+/-$ or $?$ & Doubtful reaction \\
\hline+ & Light erythema, non-vesicular \\
\hline++ & Edema, erythema, discrete vesicles \\
\hline+++ & Coalescing vesiculobullous papules \\
\hline
\end{tabular}

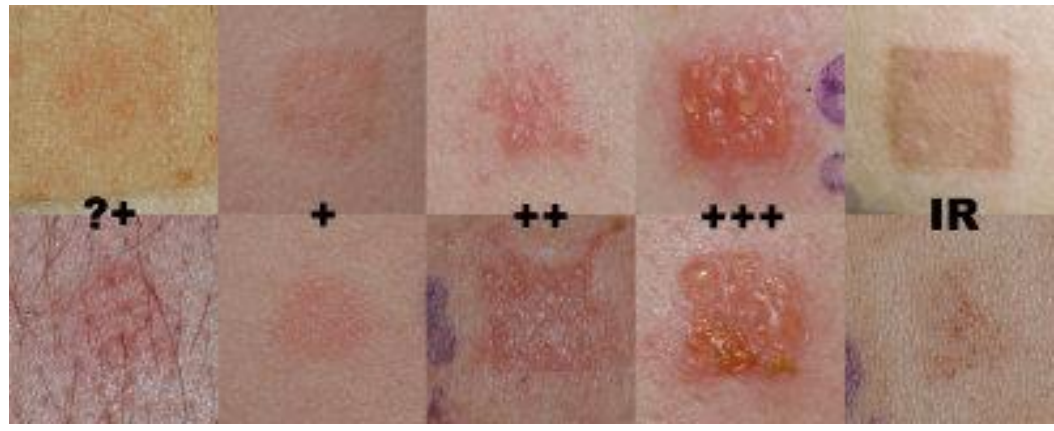

Fig. 2. A visual scheme for the interpretation of the test result [12]

Common problem occurring during patch test results evaluation is connected with interpretation of lesions as irritant, doubtful and weak reaction, especially at second reading, when uncertain grades are not allowed. In practice the difference between doubtful and weak reaction at initial reading is that if it is a weak reaction and it is negative at second reading it is an irritant allergen and then the prevention is to avoid contact to reduce symptoms like rash and red skin, and if reaction is doubtful and at another reading it is negative then patient is not allergic to that allergen. The issue is that in case of negative grade at second reading it matters if interpretation of reaction at first reading was either doubtful $(+/-)$ or weak (+). Wrong diagnostics extends time of final diagnosis, generates costs by necessity of another physical and causes discomfort of a patient.

\section{Diagnosing with the use of thermography technique}

In this chapter the measurement procedure is presented. It will be used for further improvement of objective identification of dermatitis in patch tests. The procedure of measurement for initial reading is shown on the diagram at Fig. 3 Proceedings in second and third (if was performed) reading is without strips removal.

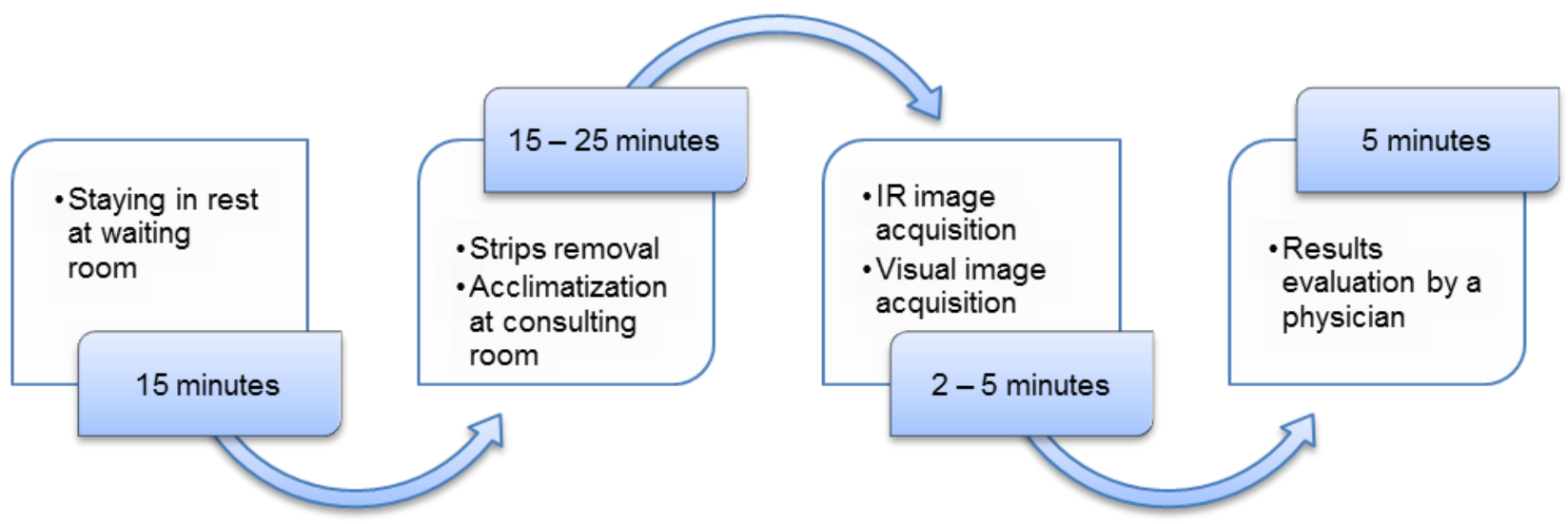

Fig. 3. Diagram of measurement procedure of initial reading after 48 hours 
Patients were examined within proposed measurement procedure at the Medical Centre Med-All in Krakow, during standard patch tests. Reading were performed after two and four days after application. In some cases additional reading was held after 7 days.

\subsection{Materials}

Thermal images were acquired by either FLIR i7 or FLIR A300 thermal imaging camera. i7 version has IR resolution $140 \times 140$ pixels, field of view (FOV) $29^{\circ} \times 29^{\circ}$, spatial resolution (IFOV) 3,7 mrad and thermal sensitivity less than $0,1^{\circ} \mathrm{C}$. A300 version has IR resolution $320 \times 240$ pixels, field of view $25^{\circ} \times 18,8^{\circ}$ and thermal sensitivity less than $0,05^{\circ} \mathrm{C}$ at $30^{\circ} \mathrm{C}$. Both versions uses uncooled microbolometer detector technology with focal plane array (FPA) and works at spectral range from 7,5 to $13 \mu \mathrm{m}$. [11]

For patch test one of used chambers were IQ Ultra by Chemotechnique Diagnostics. Those chambers are supplied in units of 10 chambers on a tape which is made from a hypoallergenic non-woven adhesive material and which is protected by a stiff plastic cover with 10 compartments that correspond to the chambers on the tape. Another used chambers, Finn Chambers, were supplied in units of one chamber on a strip, which are made of aluminium and mounted on hypoallergenic tape. [12][13]

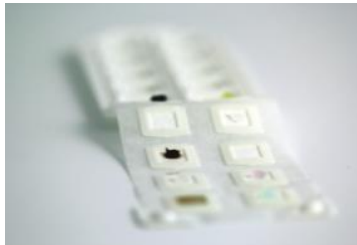

a)
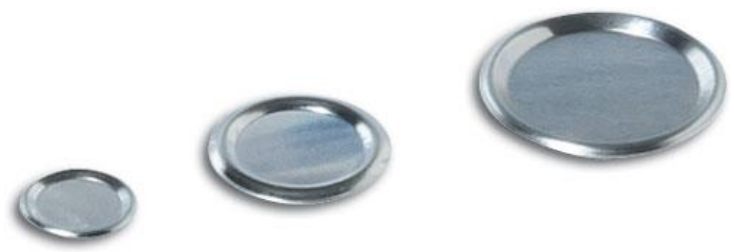

b)

Fig. 4. Chambers: a) IQ Ultra, b) Finn Chambers [12][13]

\subsection{Monitor routine}

Tests were proceeded on group of 48 patients (23 females, 8 males, aged 16-69 years and 17 children under 16 years). Among this group only 3 individuals had earlier diagnosed allergic contact dermatitis. All participants provided written informed consent. The study was performed for 16 months between 2013 and 2014.

\subsubsection{Terms and conditions at consulting room}

Measurements were carried out in a consulting room with ambient temperature within $20-21^{\circ} \mathrm{C}$, air humidity within 50-55\%, lack of external heat and radiation sources, no draught and right space. Distance between camera and subject was equal about $1 \mathrm{~m}$, because then atmospheric influence can be neglected and this distance was appropriate for chosen infrared cameras. IR camera were positioned perpendicularly to the examined surface, emissivity were settled at 0,98 and temperature scale were fixed to the patient.

\subsubsection{Preparation of a patient}

Patients were instructed that strips have to stay dry and that it is not allowed to scratch at application surface, take any cortisone medication and exposure of the back to the sun. To get valuable thermographic data the temperature of examined skin had to be stabilized before readings. That is why patients were performed acclimatization procedure, which was as follows: come calmly to the medical centre, at waiting room stay in rest for 15 minutes, in case of first reading after strips removal wait about 15-25 minutes with parts of the body which were irritated undressed, after initial reading do not scratch, wash or apply any ointment to one's surface of test application on the skin until next reading, unless doctor decides otherwise. In case of another readings, procedure is the same with the exception of the fact that strips were removed previously.

\subsection{Data analysis}

Data analysis was performed with the use of ThermoAnalysis software, MONIT SHM. It is dedicated software for acquisition and analysis of infrared images. It is great tool for advanced image processing and analysis to extract diagnostic features. Operator may perform geometric analysis (time history plots, area observation), create 3D representation of a structure and use linear and non-linear processing functions. Advanced post-processing functions enable to work with single images or a group of images at the same time. Medical diagnostics is controlled and grouped in the projects with the possibility to create IR processing timestamp which allows to create different sections with subsequent processing techniques and parameters. Thus provide to plan many diagnostic scenarios for acquired IR data and lead to compare various processing algorithms. 


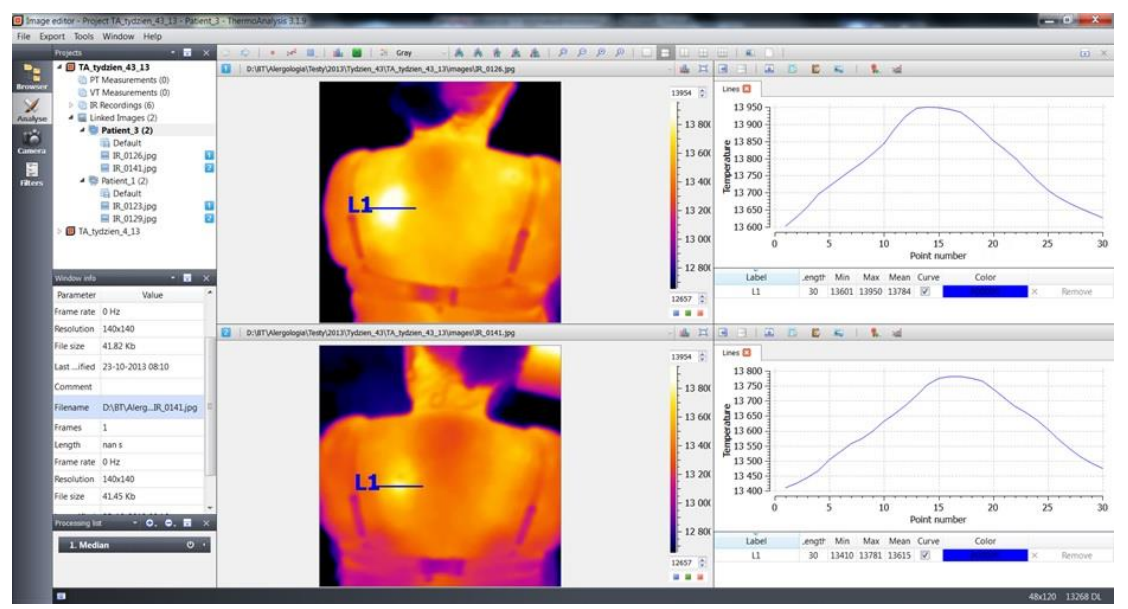

Fig. 5. Example of the medical IR data processing and analysing in ThermoAnalysis software (MONIT SHM Sp. z o.o.)[16]

\section{Medical examination}

At figures below patch test results of three representative cases are presented.

The first patient (Fig. 6 - Fig. 8) with earlier diagnosed allergic contact dermatitis to applied allergen had single chamber on the arm. Visual evaluation of physician at initial reading was negative reaction (-), and at second reading extreme reaction $(+++)$, while at thermograph positive result is well visible at both readings. It is superb visible example of delayed reaction. In this case visual symptoms were visible after 4 days, but it might last longer.

a)

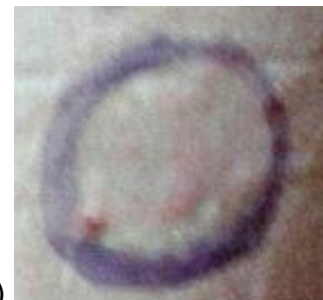

b)

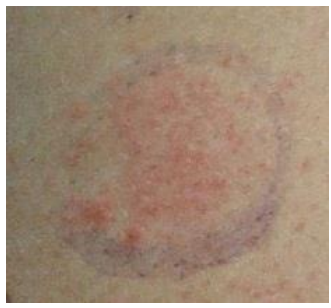

Fig. 6. Example of delayed reaction. Visual grade: a) negative reaction after 2 days and b) extreme reaction after 4 days

a)

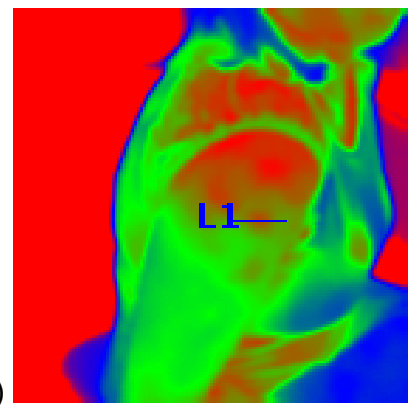

b)

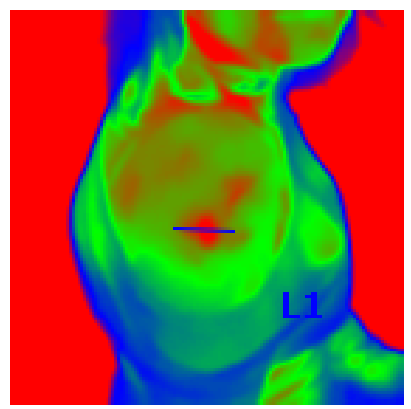

Fig. 7. Example of delayed reaction. Thermograph after: a) 2 days and b) 4 days 
a)

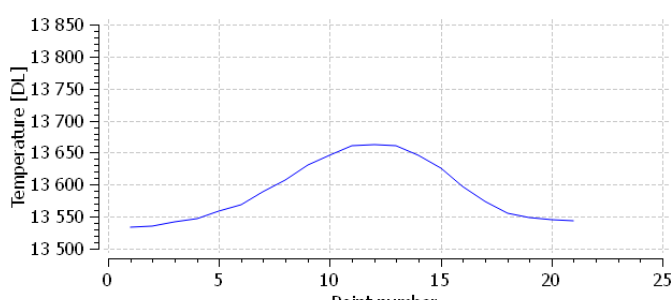

Fig. 8. Temperature changes (in digital units) at L1 section of area after incubation after: a) 2 days and b) 4 days

Second patient (Fig. 9 - Fig. 11) had extreme reaction at two allergens. Physican graded alergens with number 9 and 10 as +++ at first reading, and the same at another reading (Fig. 9). At thermograph evaluation was more difficult because thermal reaction of the skin was so huge that it covered almost half of skin surface which was incubated (Fig. 10, Fig. 11). There is no doubts that the patient is allergic to those two allergens but there is no certainty that he is not allergic to allergens from neighbour chambers.

a)

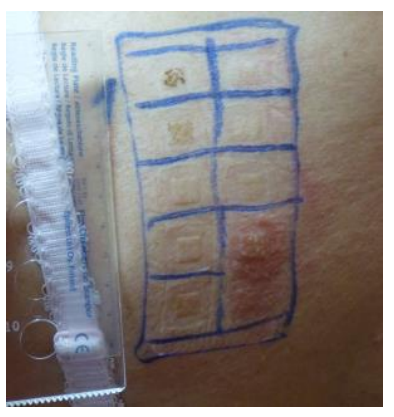

Fig. 9. Example of extreme reaction after: a) 2 days and b) 4 days

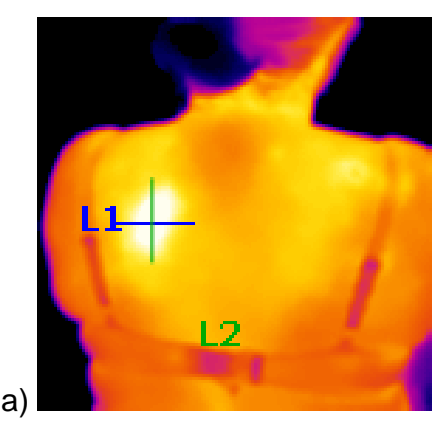

b)

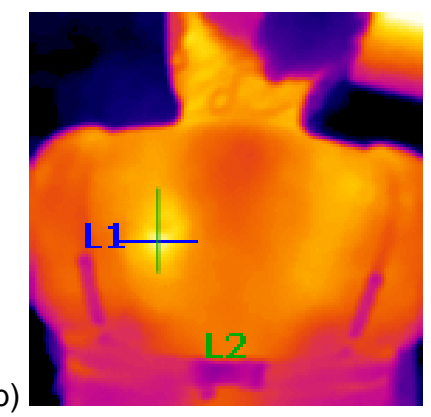

b)

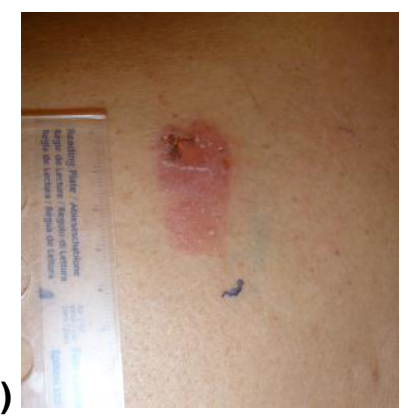

Fig. 10. Example of extreme reaction. Thermograph after: a) 2 days and b) 4 days

a)

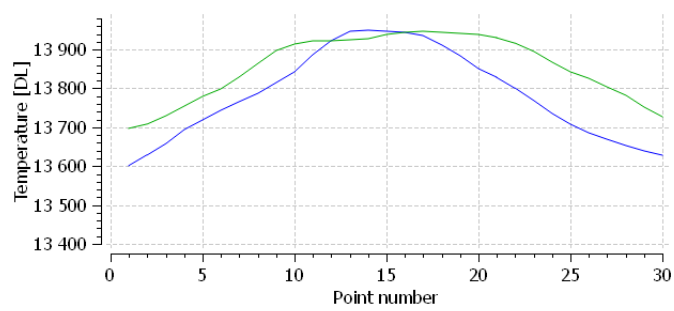


Fig. $13 \mathrm{a}$ ) and Fig. 14 a). At second reading it is hard to extract thermal reaction for allergen because it seems like the aclimatization at consulting room was too short.
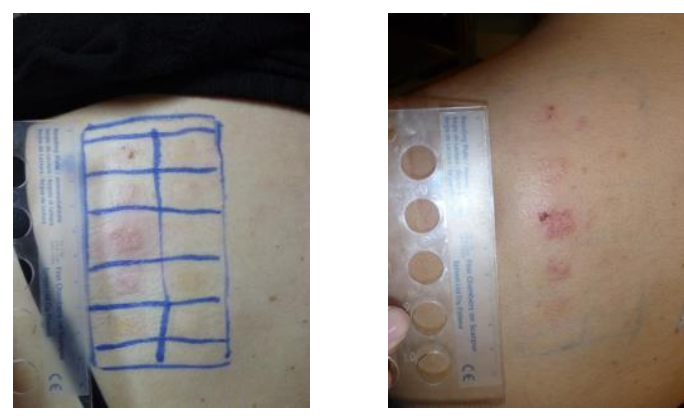

Fig. 12. Example of well visible reactions after: a) 2 days and b) 4 days
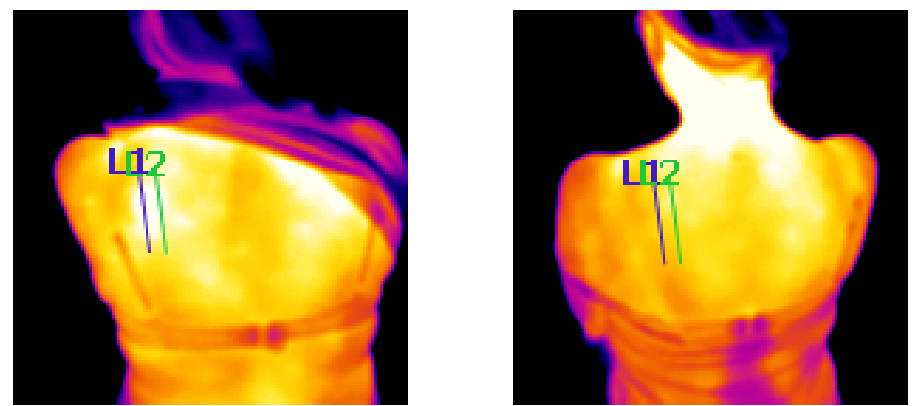

Fig. 13. Example of well visible reaction at thermograph after: a) 2 days and b) 4 days
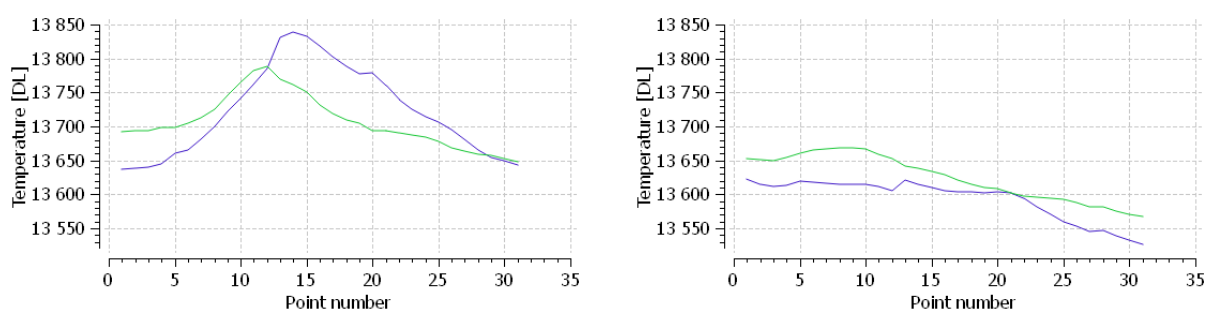

Fig. 14. Temperature changes (in digital units) at $L 1$ and $L 2$ section after: a) 2 days and b) 4 days

Table 2. Comparison of patch test results evaluation: physical and thermography

\begin{tabular}{|c|c|c|c|c|}
\hline \multirow{2}{*}{ Alergen } & \multicolumn{2}{|c|}{ First reading } & \multicolumn{2}{c|}{ Second reading } \\
\cline { 2 - 5 } & Physician & Thermograph & Physician & Thermograph \\
\hline 1 & + & & ++ & \\
\hline 2 & ++ & & +++ & \\
\hline 3 & +++ & + & +++ & + \\
\hline 4 & + & + & +++ & \\
\hline 5 & $+/-$ & + & + & \\
\hline 6 & $+/-$ & + & - & \\
\hline 7 & $+/-$ & + & - & \\
\hline 8 & - & & - & \\
\hline 9 & + & + & - & \\
\hline 10 & - & & - & \\
\hline
\end{tabular}

In medical diagnosis it is necessary to strictly define lesions, as it is shown at Table 1 (chapter 2.2). When evaluation is performed with the use of thermographic techniques, identification of lesions is made on the basis of local thermal field disturbance and, what is important, in some cases it is impossible to define dermatitis only on the basis of thermograph. In such cases, for further research, it is planned to use multimodal image processing technique, which 
effects in synergy between observation and analysis of infrared data and vision data. It enables to introduce another stage to improve objectivity of dermatitis assessment.

At Table 2 in columns Thermograph evaluation of thermal changes of patch test are presented. Empty cells at that table indicates parts of skin surface where multimodal image processing is necessary for proper thermal identification of a dermatitis.

\section{Conclusions}

Main conclusion from carried out tests is that described method is effective and when standards are adhered it gives valuable results. It is important to keep proper intervals between chambers with allergens, because thermal positive reaction to allergen is generally larger than the area of incubation on the skin while skin reaction never exceeds this area and when strong reaction occurs thermal response might cover weak reaction from neighbour allergens. Thermal stabilization is important as well, because sweaty skin on the back also covers thermal reaction to allergens. It was observed that the problem of sweat is not so significant in case of application on the arms.

What follows from this research will be used to build dedicated measurement stand and for further standardization of reading procedure. Another conclusion is that thermal imaging camera used for this application should have nearer focus range and smaller field of view because it enables more precise analysis of examined skin surface. The geometric calibration of infrared thermography system is needed to remove lens distortion from acquired IR images. For further research it is planned to create network-centric system to storage thermographs with physician description (diagnosis), case record, diagnostic results and description parameters. Those thermal images will be classified due to type of diagnosed pathology.

Medical thermal image processing is field of study which should be developed in the future, because it may significantly improve on skin tests assessment. The acquired images later will be analyzed in ThermoAnalysis software, with the use of: (1) Thermographic Signal Reconstruction (TSR) to reduce noises on the acquired images, (2) Pulsed Phase Thermography (PPT) to precise recover the gradient distribution of the thermal changes. It can be a valuable additional method in allergology, however it cannot substitute standard visual evaluation of patch tests. Presented results are first stage of longer research process.

\section{REFERENCES}

[1] Lahiri B.B., Bagavathiappan S., Jayakumar, Philip J., "Medical applications of infrared thermography: A review", Infrared Physics \& Technology, vol. 55, pp. 221-235, 2012

[2] Laino L., di Carlo A., "Telethermography: an objective method for evaluating patch test reactions", Dermatology, vol. 20, No. 2, pp. 175-180, 2010

[3] Szentkuti A., Kavanagh H.S., Grazio S., "Infrared thermography and image analysis for biomedical use", Periodicum biologorum, vol. 113, no 4, pp. 385-392, 2011

[4] Diakides N.A., Bronzino J.D., ed., "Medical Infrared Imaging", CRC Taylor\&Francis, 2008

[5] Beltrani V.S., Bernstein I.L., Cohen D.E., Fonacier L., "Contact dermatitis: a practice parameter", Annals of allergy, asthma \& immunology, vol. 97, 2006

(http://www.aaaai.org/Aaaai/media/MediaLibrary/PDF\%20Documents/Practice\%20and\%20Parameters/contact_ dermatitis_-2006.pdf)

[6] Ring E.F.J., Ammer K., "Infrared thermal imaging in medicine”, Physiological Measurement, vol. 33, pp. R33R46, 2012

[7] Ring E.F.J., Mcevoy H., Jung A., Zuber J., Machin G, "New standards for devices used for the measurement of human body temperature", Journal of Medical Engineering \& Technology, vol. 34, no. 4, pp. 249-253, 2010

[8] http://www.icdrg.org (visited 23.04.2014)

[9] ISO/TR 13154:2009 Medical electrical equipment-deployment, implementation and operational guidelines for identifying febrile humans using a screening thermograph

[10] IEC 80601-2-59:2008, Ed. 1.0 Medical electrical equipment-part 2-59: particular requirements for the basic safety and essential performance of screening thermography for human febrile temperature screening

[11] http://www.flir.com (visited 23.04.2014)

[12] http://www.chemotechnique.se (visited 23.04.2014)

[13] http://finnchamber.com (visited 23.04.2014)

[14] Vollmer M., Möllmann K.-P., "Infrared thermal imaging. Fundamentals, research and applications", WILEY-VCH Verlag GmbH \& Co. KGaA, 2010

[15] Gavriloaia G., Gavriloaia M.-R., "Improving quality of medical infrared images by using bioheat equation", Proccedings of the $3^{\text {rd }}$ International Conference on E-Health and Bioengineering - EHB 2011

[16] www.monithshm.pl 